\title{
Editorial
}

\section{Salud laboral y salud mental: estado de la cuestión}

\section{Occupational health and mental health: state of the art}

\author{
Carlos Mur de Víu ${ }^{1}$, Jerónimo Maqueda Blasco ${ }^{2}$ \\ 1. Hospital José Germain. Leganés (Madrid) España. \\ 2. Escuela Nacional de Medicina del Trabajo. Instituto de Salud Carlos III. Madrid. España.
}

\author{
Correspondencia: \\ Carlos Mur de Viu \\ Hospital José Germain \\ La Luna, 1 \\ 28991 Leganés (Madrid).España \\ Tfno: 915866767 \\ E-mail: carlos.mur@salud.madrid.org
}

Resumen

Es clave la importancia de las condiciones de trabajo en el desarrollo saludable de la persona, o como factor de riesgo psicopatológico que al aumentar el estrés hasta un rango patológico puede contribuir a que desarrolle diferentes trastornos mentales y adictivos. La enfermedad relacionada con el trabajo resulta de dos factores: Un factor individual de predisposición constitucional, y un factor profesional que ejerce una influencia favorecedora en relación con las condiciones de trabajo. Existen varios mecanismos etiopatogénicos posibles entre el hecho de padecer un trastorno mental y la actividad laboral: La exposición a estrés laboral relacionado con la falta de oportunidad para utilizar las propias capacidades da lugar a fatiga y a varios trastornos mentales, como los adaptativos, así como varias enfermedades cardio y cerebro vasculares. Por otra parte, la exposición a los riesgos psicosociales del trabajo pueden contribuir al desencadenamiento de un trastorno mental para el que el trabajador es vulnerable y el riesgo psicosocial del trabajo interviene como factor desencadenante o agravante.

Palabras clave: estrés, enfermedades psicosomáticas, trabajo, trastorno mental.

\begin{abstract}
It is a true key the importance of the work conditions in the healthy development of a person, or as a psychopathological risk factor which might contribute to the arise of addictive and mental disorders. The illness related to the work is a result of two factors: an individual factor of constitutional predisposition and a professional factor which means a favouring influence linked to the work conditions. There are different etiological mechanisms between the fact of suffering a mental disorder and the working activity: the exposition to work stress related to the lack of opportunity to use each one's capacity leads to fatigue and mental or cardiovascular diseases. On the other hand, being exposed to the psychosocial risks at work may lead to the irruption of a mental disorder to which the worker is vulnerable.
\end{abstract}

Key Words: stress, psychosomatic illnesses, work, mental disorder. 
El trabajo tiene un papel central en la vida de la mayor parte de las personas adultas, y es también una construcción social y un pilar fundamental de la sociedad humana. Una actividad laboral satisfactoria contribuye a dar sentido a la propia existencia, mejorar la salud y la autoestima, las relaciones sociales y el estatus socioeconómico. Por el contrario, unas condiciones negativas de trabajo o la ausencia del mismo, con escasa satisfacción, falta de influencia y de control en la toma de decisiones sobre las condiciones y el contenido de la tarea realizada pueden asociarse con efectos indeseables para la salud de las personas, según varios factores: el tipo de empresa, la estructura y el modelo de la organización, el estilo de dirección, los roles y el estatus del empleado, etc. Estos factores median en elevado estrés, baja satisfacción laboral y mala calidad de vida, reconocidos factores de riesgo para la salud mediadores en varios trastornos mentales y enfermedades cardiovasculares.

En los últimos años se ha evidenciado la importancia de las condiciones de trabajo en el desarrollo saludable del adolescente y del adulto al aumentar su eficacia, o como factor de riesgo psicopatológico que al aumentar el estrés hasta un rango patológico puede contribuir a que desarrolle diferentes trastornos mentales y adictivos. Ambas posibilidades dependen de las características de las condiciones de trabajo, como del número de horas de trabajo a la semana, de la calidad de la supervisión, y de que facilite o interfiera en la realización de otras tareas como en el aprendizaje de nuevos conocimientos y la adquisición de habilidades. Un trabajo rutinario y agotador con una supervisión inadecuada, que media en un elevado nivel de estrés, puede contribuir a desarrollar varios tipos de enfermedades.

Hay tres efectos básicos que enlazan las interacciones complejas que tienen lugar entre empleo y salud mental de modo circular, tanto en sentido positivo como negativo. Estos son los efectos del empleo/ desempleo sobre la salud mental, los de la enfermedad mental sobre el empleo, y los de otros factores que modifican ambas variables tales como el estatus marital, la existencia de hijos en el hogar y la clase social, que deben ser controlados simultáneamente. En general el hecho de tener un empleo protege la salud mental de hombres y mujeres, salvo por sobrecarga de trabajo y conflicto de roles empleo/familia. Incluso los pacientes con esquizofrenia que pueden mantener el empleo muestran menor nivel de ansiedad y mayor nivel de iniciativa y de actividad. El trabajo puede intervenir como causa o agravante a través de tres factores fundamentales: condiciones del medio ambiente material, medio ambiente psicológico y medio ambiente socioeconómico.

La enfermedad relacionada con el trabajo resulta de dos factores: Un factor individual de predisposición constitucional, y un factor profesional que ejerce una influencia favorecedora en relación con las condiciones de trabajo.

El trabajo puede desencadenar y/o agravar una enfermedad compleja y de etiología multifactorial para la que el paciente está predispuesto, como la hipertensión arterial esencial y varios trastornos mentales como la depresión y las adicciones. Las enfermedades en el ámbito laboral pueden desencadenarse por diferentes condiciones de trabajo como son: características generales de locales, instalaciones, equipos, productos y demás útiles en el centro de trabajo; la naturaleza de agentes físicos, químicos y biológicos en el ambiente de trabajo y sus intensidades, concentraciones o niveles de presencia; los procedimientos utilizados en la realización de las tareas; la dedicación horaria; y otras características del trabajo, como la organización y ordenación, que influyen en la magnitud de los riesgos a que está expuesto el trabajador, cuando no se aplican correctamente los principios de la Ergonomía.

Existen varias trayectorias o mecanismos etiopatogénicos posibles entre el hecho de padecer un trastorno mental y la actividad laboral según la evidencia científica actualmente disponible:

1. ${ }^{\circ}$ La exposición a factores psicosociales de riesgo del trabajo, como el estrés laboral relacionado con la falta de control y de oportunidad para utilizar las propias capacidades, que se comportan de manera acumulativa negativa, disminuyen el rendimiento en la realización de la tarea y dar lugar a un estado de fatiga y a varios trastornos mentales, como los adaptativos, así como varias enfermedades psicosomáticas relacionadas con el estrés como las cardio y cerebrovasculares, dolor lumbar y bajas laborales, con independencia de las características psicológicas de las personas estudiadas. La deprivación material en sí misma produce conductas de riesgo para la salud de tipo nutricional, sedentarismo y conductas adictivas. El estrés del trabajo se ha convertido en una de las principales causas de incapacidad laboral en los países desarrollados, al mediar en "nuevas y sutiles amenazas para la salud mental" y como factor de riesgo de enfermedades como las cardiovasculares, los trastornos mentales y los accidentes de trabajo. Se ha constatado que el estrés en el trabajo tiene un 
importante papel mediador en las grandes diferencias en salud, enfermedad y muerte prematura, relacionadas con el estatus sociolaboral.

2. ${ }^{\circ}$ La exposición a los riesgos psico-sociales del trabajo pueden contribuir de forma inespecífica al desencadenamiento de un trastorno mental para el que el trabajador es vulnerable y el riesgo psicosocial del trabajo interviene como factor desencadenante o agravante, como en el caso de los trastornos depresivos y de ansiedad, sobre todo en los primeros episodios. Se atribuye a las situaciones estresantes el $\mathbf{1 0 - 1 5 \%}$ de la varianza sobre la sintomatología depresivo-ansiosa que padece el paciente. Se trata de trastornos de etiología multifactorial, de forma que cada factor explica una proporción relativamente pequeña de la varianza, lo que obliga a tener en cuenta diferentes factores de vulnerabilidad o modificadores, genéticos, evolutivos, psicológicos y sociales.

3. ${ }^{\circ}$ De forma específica la exposición a situaciones laborales estresantes traumáticas, como accidentes laborales graves y acoso en el trabajo pueden causar patologías relacionadas con el estresor traumático, factor necesario pero no suficiente, que produce trastornos como el trastorno de estrés postraumático, ya reconocido como enfermedad profesional cuando se produce tras la exposición a experiencias traumáticas en el trabajo.

Las Condiciones de Trabajo, como determinantes del estado de salud de la persona, son por lo tanto indisociables de otros determinantes tanto intrínsecos como extrínsecos y éstas, a su vez, son dependientes del momento de desarrollo económico y modelo productivo.

Los procesos de innovación tecnológica, la evolución de los mercados de trabajo y la emergencia de nuevas actividades han transformado la relación Trabajo/ Salud, han generado una nueva epidemiología de la enfermedad en el trabajo. En esta nueva epidemiología el "factor externo" evidenciable en forma de una máquina, una sustancia cancerígena, sensibilizador o irritante ha perdido su hegemonía en el complejo causal de la enfermedad. Complejo que se ha desplazado hacia componentes de "sobre carga" de funciones tanto biológicas como mentales y sociales.

En la actual Sociedad del Trabajo los elementos desestabilizadores de la salud se ubican, principalmente, en la esfera de la "adaptación" y la "relación". El profesional de la prevención debe adquirir nuevas habilidades en la identificación, manejo e intervención sobre una "enfermedad" para la que habitualmente no está suficientemente entrenado, buscando alianzas con el ámbito de la salud mental.

Por tanto, podemos concluir que la interrelación entre la salud laboral y la salud mental es cada vez más definida y estudiada. El propósito de este monográfico no es otro que el de ampliar la información y reflexión sobre este tema de gran actualidad de mano de grandes expertos de distintas disciplinas. El abordaje multidisciplinar desde distintos puntos de vista (médicos del trabajo, médicos psiquiatras, psicólogos clínicos, trabajadores sociales...) nos aporta una visión sinérgica y completa de un campo tan apasionante y de actualidad como los trastornos mentales en el ámbito laboral. 